\title{
TRANSITION DENSITY ESTIMATES FOR RELATIVISTIC $\alpha$-STABLE PROCESSES ON METRIC SPACES
}

\author{
BY \\ HUBERT BALSAM (WARSZAWA) AND \\ KATARZYNA PIETRUSKA-PAŁUB ** (WARSZAWA)
}

\begin{abstract}
We prove matching upper and lower bounds for the transition density of relativistic $\alpha$-stable processes on a $d$-set $(F, \rho, \mu)$, obtained via subordination. We also identify the corresponding Dirichlet form.
\end{abstract}

2020 Mathematics Subject Classification: Primary 60J35, 60J76; Secondary 60B99.

Key words and phrases: relativistic stable process, $d$-set, transition density, Dirichlet form.

\section{INTRODUCTION}

The theory of stochastic processes on irregular sets, such as fractals, has been rapidly developing since the 1980's. The first to be constructed was the Brownian motion on the Sierpiński gasket [5], on the Sierpiński carpet [2], on more general nested fractals [36], [33], [17], on post-critically finite sets [33], and also in more general contexts [43]. In fractal cases, these processes fall within the framework of diffusions on $d$-sets consistent with the geometric structure of the state space, and typically are unique up to a linear time-change [39], [4]. For a general account of such processes we refer to [1]. In all these cases, the constructed process is a strong Markov process on the underlying metric space $(F, \rho, \mu)$ with continuous trajectories, which gives rise to a heat kernel on $F$ with two-sided sub-Gaussian estimates:

$$
\begin{aligned}
C_{1} t^{-d / d_{w}} \exp \{ & \left.-C_{2}\left(\rho(x, y) t^{-1 / d_{w}}\right)^{\frac{d_{w}}{d_{w}-1}}\right\} \\
& \leqslant g(t, x, y) \leqslant C_{3} t^{-d / d_{w}} \exp \left\{-C_{4}\left(\rho(x, y) t^{-1 / d_{w}}\right)^{\frac{d_{w}}{d_{w}-1}}\right\},
\end{aligned}
$$

where $d_{w}$ is the so called walk dimension of $F$, and $C_{1}, \ldots, C_{4}>0$ are certain constants.

\footnotetext{
${ }^{*}$ Supported by the NCN grant 2014/14/M/ST1/00600.
} 
The generator of this process is called the Laplacian on $F$, although it does not have a straightforward differential meaning, even for fractal subsets of the Euclidean space-e.g. for the Laplacian on the Sierpiński gasket, no function in its domain has a $C^{1}$ extension to any open neighbourhood of the gasket (see [5, Corollary 9.3]).

Diffusion processes on irregular sets became popular in connection with the theory of disordered media, especially processes that evolve at percolation clusters at criticality, which are expected to exhibit multi-level self-similarity. For a fair account of the physics literature on the subject we refer to [21], and for mathematical aspects to [1]. In this vein, also discontinuous processes with values in a general metric measure space (required to have some regularity properties) have been considered. So far, the best analysed are the $\alpha$-stable processes on $d$-sets (not necessarily embedded in the Euclidean space) defined independently in [11] and [14]. The generator of the $\alpha$-stable process corresponds to the operator $-(-\Delta)^{\alpha}, \alpha \in(0,2)$, on $\mathbb{R}^{d}$, i.e. the ultra-relativistic Hamiltonian. From the mathematical point of view, a natural step forward is to consider relativistic Hamiltonians $-\left(-c \Delta+m^{1 / \alpha}\right)^{\alpha}+m, m>0$, which are important approximations of ultra-relativistic quantum mechanics. In particular, when $\alpha=1 / 2$, the Hamiltonian $\sqrt{-\hbar c^{2} \Delta+m^{2} c^{4}}$ (called the Klein-Gordon square root operator or the quasi-relativistic Hamiltonian) is often used to describe the motion of a free quasi-relativistic particle. Here $m$ is the mass of the particle, $c$ is the speed of light, and $\hbar$ is the reduced Planck constant. Since the term $m c^{2}$ represents the rest mass, the related operator $-L:=\sqrt{-\hbar c^{2} \Delta+m^{2} c^{4}}-m c^{2}$ is also called the kinetic energy operator. This theory has been strongly influenced by Lieb and Seiringer's investigations on the stability of (relativistic) matter [35].

In this paper, we propose to define a relativistic $\alpha$-stable process on a given measure metric space $(F, \rho, \mu)$ via Bochner subordination of the Brownian motion on $F$. This classical procedure, developed by Bochner [8], [9], gives a tool for constructing new semigroups (operators, processes) from a given one. For a more recent account on subordination, we refer to [6], [7], [40]. See also Section 2.2 below for a more detailed description. A particular feature of Bochner subordination is that it preserves functional inequalities, e.g. Nash and Poincaré inequalities (see [41]), which can be further related to properties of the semigroup such as on-diagonal estimates of the kernel (see e.g. [12]).

Bochner subordination was used in the pioneering paper [16] to define traces of processes subordinate (via stable subordinators) to general diffusions in a domain $\Omega \subset \mathbb{R}^{n}$ to its irregular boundary $\partial \Omega=\Gamma$. This boundary is required to be a $d$-set with $n-1 \leqslant d<n$. The authors proved that the subordinate process on the domain $\Omega$ yields a Dirichlet form on its boundary $\Gamma$, which is a generalization of similar results from [26], which were proved for a diffusion and a domain with smooth boundary.

In the present paper, the situation is somewhat different. We do not require the set to be embedded in the Euclidean space, and even for those that are, we consider 
an intrinsic metric on the set, which may be incomparable with the Euclidean one. We start with the Brownian motion on the already irregular space (an abstract one, not necessarily the boundary of a domain in $\mathbb{R}^{n}$ ), and as a result we obtain a process on the same irregular space. Stable subordinators were introduced in this context in [11], and now we work with closely related, but more complicated, relativistic subordinators.

Simultaneously, in parallel to the stochastic considerations coming mostly from mathematical physics, there has been a vivid interest in the theory of function spaces on irregular sets. Initially, this theory was developed for sets embedded in $\mathbb{R}^{n}$, including fractals (see [29], [45], [44] and the literature therein). In particular, Besov spaces were introduced and analysed in this generality. They were naturally linked to diffusions on fractals: the domain of the Dirichlet form of the Brownian motion turned out to be a Besov space (see [27] for the Sierpiński gasket, and [37] for $d$-sets). In this direction, function spaces on fractals and more general measure metric spaces were systematically analyzed (see e.g. [24], [25], [34], [19]). We also point out two papers concerned with trace theorems: [28] on the Sierpiński gasket and [23] on general fractals.

Properties of subordinate $\alpha$-stable processes on $d$-sets, including transition density estimates, were analysed in [11], [42] by Bogdan, Stós and Sztonyk. Following that path, in this paper we give an elementary proof of two-sided estimates for the transition density of a relativistic $\alpha$-stable process on a $d$-set (Theorems 3.1 and 3.2), and then we identify the Dirichlet form of the process (Proposition 3.1). To get our estimates, we just use subordination and some known properties of $\alpha$ stable subordinators.

More precisely, denoting by $p(\cdot, \cdot, \cdot)$ the transition density of a subordinate relativistic $\alpha$-stable process on a $d$-set $(F, \rho, \mu)$, we prove that

(1) for $t \geqslant 1$ and $x, y \in F$,

$$
p(t, x, y) \asymp C_{*} t^{-d / d_{w}} \exp \left\{-C_{*} \min \left(\rho(x, y),\left(\rho(x, y) t^{-1 / d_{w}}\right)^{\frac{d_{w}}{d_{w}-1}}\right)\right\},
$$

(2) for $t \in(0,1)$ and $x, y \in F$ with $\rho(x, y) \geqslant 1$,

$$
p(t, x, y) \asymp C_{*} t \exp \left\{-C_{*} \rho(x, y)\right\},
$$

(3) for $t \in(0,1)$ and $x, y \in F$ with $\rho(x, y)<1$,

$$
p(t, x, y) \asymp C_{*} \min \left(t \rho(x, y)^{-d-\alpha d_{w}}, t^{-d /\left(\alpha d_{w}\right)}\right),
$$

where $C_{*}$ denotes a constant that can be different in the upper and lower bounds. These estimates are consistent with those for relativistic stable processes in $\mathbb{R}^{d}$ from [14] (see (1.1) below), with parameters adjusted to the present setting. We also mention that in the case of the Euclidean space $\mathbb{R}^{d}$, it is possible (see [30]) to 
obtain bounds with the same upper and lower exponent in the regime (2) above (a polynomial correction is needed outside the exponent in that case).

When the 'jumping intensity' $J$ of the process can be prescribed a priori, the process can be described starting from its Dirichlet form. This approach was pursued by Chen and Kumagai [14] to prove estimates for $\alpha$-stable processes on $d$-sets, and then for more general processes in [15]. In particular, as an application of their method, the authors consider a relativistic process in $\mathbb{R}^{d}$ (not on general $d$-sets) and for $t>1$ they obtain the estimate

$$
p(t, x, y) \asymp C_{*} t^{-d / 2} \exp \left\{-C_{*} \min \left(|x-y|,|x-y|^{2} / t\right)\right\} .
$$

(For small $t$ 's, their estimate is similar to that for $\alpha$-stable processes.)

In the general situation, it was not clear what kernel $J$ should correspond to a metric-space counterpart of a relativistic process on $\mathbb{R}^{d}$. The approach of this paper permits us to identify the kernel (or at least to give an upper and a lower bound) on general $d$-sets. The bounds on the jumping kernel are derived directly from the estimates on the transition density.

The paper is organized as follows. In Section 2 we provide some definitions and notations regarding fractional diffusions, subordination and Dirichlet forms. Section 3 contains the proof of the main theorems, on estimates of the transition density. We finish this section by determining the domain of the Dirichlet form of the process and proving estimates on the kernel of this form.

\section{PRELIMINARIES}

Notation. Throughout the paper, upper- and lowercase numbered constants, $A_{i}$, $K_{i}, C_{i}, c_{i}$, denote constants whose values, once fixed, will not change. The constants that are not numbered, i.e. $c, C, c^{\prime}, C^{\prime}, \ldots$, can change their value inside the proofs. For two functions defined on a common domain, $f \asymp g$ means that there is an absolute constant $C>0$ such that $\frac{1}{C} f(\cdot) \leqslant g(\cdot) \leqslant C f(\cdot)$.

2.1. Fractional diffusion, $d$-set and $d$-measure. In this section we introduce some definitions and notation taken from [1], [29], as well as some formulas used later.

Let $(F, \rho, \mu)$ be a locally compact, separable metric space and let $\mu$ be a Borel measure on $F$.

Definition 2.1. Suppose $d>0$. A positive Borel measure $\mu$ on $F$ is called a $d$-measure if there exist constants $c_{1.1}, c_{1.2}>0$ such that

$$
c_{1.1} r^{d} \leqslant \mu(B(x, r)) \leqslant c_{1.2} r^{d} \quad \forall x \in F, r \in(0, \operatorname{diam} F),
$$

where $B(x, r)=\{y \in F: \rho(x, y)<r\}$ is the open ball in $F$ in the metric $\rho$.

DEFINITION 2.2. A closed set $F$ is called a $d$-set if there exists a $d$-measure on $(F, \rho)$ with support $F$. 
All $d$-measures on a fixed set $F$ are equivalent. The Hausdorff dimension of any $d$-set is equal to $d$. For more information on $d$-sets and $d$-measures we refer to [29].

From now on we assume that $F$ is a given $d$-set equipped with a $d$-measure $\mu$. We introduce the following definition of fractional diffusion, adapted from [1]:

Definition 2.3. Let $\left(Z_{t}, \mathbf{P}_{x}\right)_{x \in F, t \geqslant 0}$ be a Markov process on $F$. We call it a fractional diffusion if:

1. $Z$ is a Feller diffusion with state space $F$,

2. $Z$ has a symmetric continuous transition density $g(\cdot, \cdot, \cdot)$ with respect to the $d$-measure $\mu$ such that

$$
\begin{aligned}
& C_{1} t^{-d / d_{w}} \exp \left\{-C_{2}\left(\rho(x, y) t^{-1 / d_{w}}\right)^{\frac{d_{w}}{d_{w}-1}}\right\} \\
& \leqslant g(t, x, y) \leqslant C_{3} t^{-d / d_{w}} \exp \left\{-C_{4}\left(\rho(x, y) t^{-1 / d_{w}}\right)^{\frac{d_{w}}{d_{w}-1}}\right\}
\end{aligned}
$$

for all $t \in\left(0,(\operatorname{diam} F)^{d_{w}}\right)$ and $x, y \in F$, with constants $C_{1}, \ldots, C_{4}>0$ and $d_{w}>1$.

The constant $d_{w}$ is called the walk dimension on $F$ and depends only on the geometry of $F$ (see [37]), i.e. for a given $d$-set $F$ all fractional diffusions on $F$ have the same parameter $d_{w}$ (the proof in [37] was given only for subsets of $\mathbb{R}^{n}$, but it works for general metric spaces, see [20]). In particular, for $F=\mathbb{R}^{n}$, we have $d_{w}=2$, and if $F$ is the Sierpiński gasket then $d_{w}=\frac{\log 5}{\log 2}$.

2.2. Subordination. Let $\left(T_{t}\right)_{t \geqslant 0}$ be a strongly continuous semigroup on $L^{p}(F, \mu)$ with generator $(A, \mathcal{D}(A))$, and let $S=\left(S_{t}, \mathbf{P}\right)_{t \geqslant 0}$ be a subordinator, i.e. an increasing Lévy process on $[0, \infty)$ such that $S_{0}=0$ (see [7]). Denote by $\eta_{t}(\mathrm{~d} s)$ the distribution of $S_{t}$; then the Laplace transform of $\eta_{t}$ can be written in the form

$$
\int_{0}^{\infty} \mathrm{e}^{-\lambda s} \eta_{t}(\mathrm{~d} s)=\mathrm{e}^{-t \phi(\lambda)}, \quad \lambda>0 .
$$

The measures $\eta_{t}, t \geqslant 0$, form a convolution semigroup on $[0, \infty)$.

The function $\phi:(0, \infty) \rightarrow \mathbb{R}$ is called the Laplace exponent of $S$ and can be expressed as (Lévy-Khinchin formula):

$$
\phi(\lambda)=a \lambda+\int_{0}^{\infty}\left(1-\mathrm{e}^{-\lambda x}\right) \nu(\mathrm{d} x)
$$

where $a \in \mathbb{R}$ is the drift coefficient of $S$, and $\nu$ is the Lévy measure of $S$; it is a nonnegative, $\sigma$-finite, Borel measure on $(0, \infty)$ which satisfies the condition

$$
\int_{0}^{\infty}(1 \wedge x) \nu(\mathrm{d} x)<\infty
$$


We define a new semigroup

$$
T_{t}^{\phi} u:=\int_{0}^{\infty} T_{s} u \eta_{t}(\mathrm{~d} s)
$$

which is again a strongly continuous semigroup on $L^{p}(F, \mu)$. The integral is understood in the sense of Bochner and is well-defined under the present assumptions. This is the semigroup subordinate to $\left(T_{t}\right)$, sometimes denoted $\left(T_{t}^{\phi}\right)$, with generator $\left(A^{\phi}, \mathcal{D}\left(A^{\phi}\right)\right)$.

In particular, when $p=2$ and $T_{t}$ is the semigroup of a Markov process $Z$ on $F$ with transition densities $g(t, x, y), t>0, x, y \in F$, then $T_{t}^{\phi}$ is also a semigroup of a Markov process $X$, called the process subordinate to $Z$ via the subordinator $S$, whose transition probabilities are given by

$$
p(t, x, A)=\int_{0}^{\infty} \int_{A} g(u, x, y) \mu(\mathrm{d} y) \eta_{t}(\mathrm{~d} u) .
$$

Formally, we have $X_{t}=Z_{S_{t}}$.

Two classes of subordinators will be important for our purposes.

2.2.1. $\alpha$-stable (jump) subordinators. Let $\alpha \in(0,1)$. We call a subordinator $S$ $\alpha$-stable if its Laplace exponent $\phi$ is

$$
\phi(\lambda)=\lambda^{\alpha}, \quad \lambda>0 .
$$

Integration by parts gives

$$
\lambda^{\alpha}=\frac{\alpha}{\Gamma(1-\alpha)} \int_{0}^{\infty}\left(1-\mathrm{e}^{-\lambda x}\right) x^{-1-\alpha} \mathrm{d} x
$$

which means that the Lévy measure of $S$ is $\nu(\mathrm{d} x)=(\alpha / \Gamma(1-\alpha)) x^{-1-\alpha} \mathrm{d} x$, and the restriction $\alpha \in(0,1)$ follows naturally from the condition 2.4$)$. In this case, for any $t>0$ the measure $\eta_{t}$ is absolutely continuous with respect to the Lebesgue measure. We will use the same letter for this measure and for its density, $\eta_{t}(\mathrm{~d} s)=\eta_{t}(s) \mathrm{d} s$. We collect the properties of $\eta_{t}$ in a lemma.

Lemma 2.1. Let $\eta_{t}$ be the density of an $\alpha$-stable subordinator, $\alpha \in(0,1)$. Then the following hold true.

(i) The scaling property: for any $t, s>0$,

$$
\eta_{t}(s)=t^{-1 / \alpha} \eta_{1}\left(t^{-1 / \alpha} s\right) .
$$

There exist constants $A_{1}, A_{2}, a_{0}, a_{1}, a_{2}>0$, either absolute or depending only on $\alpha$, such that:

(ii) Asymptotic behaviour at $0^{+}$(see [22, proof of Lemma 1]): the limit below exists:

$$
\lim _{s \rightarrow 0^{+}} \eta_{1}(s) s^{a_{1}} \mathrm{e}^{a_{2} s^{-\frac{\alpha}{1-\alpha}}}=A_{1}
$$


(iii) Asymptotic behaviour at $\infty$ (see [10, p. 97]):

$$
\lim _{s \rightarrow \infty} \eta_{1}(s) s^{1+\alpha}=A_{2} \text {. }
$$

(iv) A global estimate (see [11, formula (14)]):

$$
\eta_{t}(s) \leqslant a_{0} t s^{-1-\alpha} \mathrm{e}^{-t s^{-\alpha}}, \quad s, t>0 .
$$

2.2.2. Relativistic $\alpha$-stable subordinators. Let $\alpha \in(0,1)$ and $m>0$. A subordinator $S$ is called relativistic $\alpha$-stable with mass $m$ if its Laplace exponent is

$$
\phi(\lambda)=\left(\lambda+m^{1 / \alpha}\right)^{\alpha}-m, \quad \lambda>0 .
$$

Again, integration by parts gives

$$
\left(\lambda+m^{1 / \alpha}\right)^{\alpha}-m=\frac{\alpha}{\Gamma(1-\alpha)} \int_{0}^{\infty}\left(1-\mathrm{e}^{-\lambda x}\right) \mathrm{e}^{-m^{1 / \alpha} x} x^{-1-\alpha} \mathrm{d} x,
$$

so that in this case the Lévy measure is

$$
\nu(\mathrm{d} x)=(\alpha / \Gamma(1-\alpha)) \mathrm{e}^{-m^{1 / \alpha} x} x^{-1-\alpha} \mathrm{d} x .
$$

Denoting by $\eta_{t, m}(\cdot)$ the density of the relativistic $\alpha$-stable subordinator, we have (see [38, p. 3])

$$
\eta_{t, m}(s):=\mathrm{e}^{-m^{1 / \alpha} s+m t} \eta_{t}(s), \quad m>0, s, t>0 .
$$

Indeed, from (2.5) we verify that the Laplace transform of $\eta_{t, m}$ equals that given by 2.10$)$ :

$$
\int_{0}^{\infty} \mathrm{e}^{-\lambda s} \eta_{t, m}(s) \mathrm{d} s=\mathrm{e}^{m t} \int_{0}^{\infty} \mathrm{e}^{-\left(\lambda+m^{1 / \alpha}\right) s} \eta_{t}(s) \mathrm{d} s=\mathrm{e}^{-t\left[\left(\lambda+m^{1 / \alpha}\right)^{\alpha}-m\right]} .
$$

For more examples of subordinators and their properties we refer to [7], [6], [40].

2.3. Stable and relativistic $\alpha$-stable processes. Assume that $\left(Z_{t}, \mathbf{P}_{x}\right)_{x \in F, t \geqslant 0}$ is a fractional diffusion on $(F, \rho, \mu)$ and let $S$ be a subordinator independent of $Z$. We define a new process $X=\left(X_{t}\right)_{t \geqslant 0}$ as

$$
X_{t}:=Z_{S_{t}}, \quad t \geqslant 0 .
$$

The process $X$ is called the subordinate Brownian motion on $F$ (via the subordinator $S$ ). It is a Markov process with càdlàg paths whose transition density function can be represented as

$$
p(t, x, y)=\int_{0}^{\infty} g(s, x, y) \eta_{t}(\mathrm{~d} s) .
$$

If $S=\left(S_{t}, \mathbf{P}\right)_{t \geqslant 0}$ is an $\alpha$-stable subordinator, $\alpha \in(0,1)$, then $X_{t}:=Z_{S_{t}}$ is called an $\alpha$-stable process on $F$. Likewise, if $S=\left(S_{t}, \mathbf{P}\right)_{t \geqslant 0}$ is a relativistic $\alpha$-stable 
subordinator, then $X_{t}:=Z_{S_{t}}$ is a relativistic $\alpha$-stable process on F. From 2.11) we see that in this case the transition density is given by

$$
p(t, x, y)=\mathrm{e}^{m t} \int_{0}^{\infty} g(s, x, y) \mathrm{e}^{-m^{1 / \alpha} s} \eta_{t}(s) \mathrm{d} s
$$

where $\eta_{t}(\cdot)$ is the density of the pure $\alpha$-stable subordinator. This formula will be the starting point for our investigations.

2.4. Dirichlet forms. We finish the introductory part by sketching some definitions regarding Dirichlet forms, taken from [12]. For more details we refer to [18].

Let $p(t, x, y)$ be the transition density of a symmetric Markov process $\left(X_{t}\right)$ on $(F, \rho, \mu)$. For $f \in L^{2}(F, \mu)$ we define

$$
\mathcal{E}_{t}(f, f)=\frac{1}{2 t} \int_{F} \int_{F}(f(x)-f(y))^{2} p(t, x, y) \mu(\mathrm{d} x) \mu(\mathrm{d} y) .
$$

By spectral theory, for any $f \in L^{2}(F, \mu)$ the function $t \mapsto \mathcal{E}_{t}(f, f)$ is decreasing for $t>0$, and we set

$$
\mathcal{E}(f, f)=\lim _{t \rightarrow 0^{+}} \mathcal{E}_{t}(f, f), \quad \mathcal{D}(\mathcal{E})=\left\{f \in L^{2}(F, \mu): \mathcal{E}(f, f)<\infty\right\} .
$$

Then $(\mathcal{E}, \mathcal{D}(\mathcal{E}))$ is called the Dirichlet form of the process $\left(X_{t}\right)$. As an example, for a symmetric $\alpha$-stable process on $F, \alpha \in(0,1)$, we have (see [42])

$$
\begin{aligned}
\mathcal{D}\left(\mathcal{E}^{\text {stab }}\right) & =\operatorname{Lip}\left(\alpha d_{w} / 2,2,2, F\right) \\
& =\left\{f \in L^{2}(F, \mu): \int_{F} \int_{F} \frac{(f(x)-f(y))^{2}}{\rho(x, y)^{d+\alpha d_{w}}} \mu(\mathrm{d} x) \mu(\mathrm{d} y)<\infty\right\},
\end{aligned}
$$

and

$$
\mathcal{E}^{\mathrm{stab}}(f, f) \asymp \int_{F} \int_{F} \frac{(f(x)-f(y))^{2}}{\rho(x, y)^{d+\alpha d_{w}}} \mu(\mathrm{d} x) \mu(\mathrm{d} y) .
$$

\section{AN ESTIMATE OF THE TRANSITION DENSITY FUNCTION FOR A RELATIVISTIC $\alpha$-STABLE PROCESS}

The transition density estimates we are going to obtain are split in two parts; $t \geqslant 1$ and $t<1$. We address these two regimes separately. We start with $t \geqslant 1$.

THEOREM 3.1. Let $X_{t}$ be a relativistic $\alpha$-stable process on $F$, with density function $p(\cdot, \cdot, \cdot)$ given by (2.12). Let $t \geqslant 1$. Then there exist constants $K_{i}=$ $K_{i}(\alpha)>0, i=1, \ldots, 4$, such that for $x, y \in F$,

$$
\begin{aligned}
K_{1} t^{-d / d_{w}} & \exp \left\{-K_{2} \min \left(\rho(x, y),\left(\rho(x, y) t^{-1 / d_{w}}\right)^{\frac{d w}{d w-1}}\right)\right\} \leqslant p(t, x, y) \\
& \leqslant K_{3} t^{-d / d_{w}} \exp \left\{-K_{4} \min \left(\rho(x, y),\left(\rho(x, y) t^{-1 / d_{w}}\right)^{\frac{d_{w}}{d_{w}-1}}\right)\right\} .
\end{aligned}
$$


Proof. I. The lower bound. First we show that there exist $K_{1}(\alpha), K_{2}(\alpha)>0$ such that for any $x, y \in F$,

$$
p(t, x, y) \geqslant K_{1} t^{-d / d_{w}} \exp \left\{-K_{2} \min \left(\rho(x, y),\left(\rho(x, y) t^{-1 / d_{w}}\right)^{\frac{d_{w}}{d_{w}-1}}\right)\right\} .
$$

From the expression defining $p(\cdot, \cdot, \cdot)$,

$$
p(t, x, y)=\mathrm{e}^{m t} \int_{0}^{\infty} g(s, x, y) \mathrm{e}^{-m^{1 / \alpha} s} \eta_{t}(s) \mathrm{d} s,
$$

using subgaussian estimates on $g,(2.1)$, and scaling of $\eta_{t},(2.6)$, we get

$$
p(t, x, y) \geqslant C_{1} \mathrm{e}^{m t} \int_{0}^{\infty} s^{-d / d_{w}} \mathrm{e}^{-C_{2} s^{\frac{-1}{d_{w}-1}} \rho(x, y)^{\frac{d_{w}}{d_{w}-1}}} \mathrm{e}^{-m^{1 / \alpha} s} t^{-1 / \alpha} \eta_{1}\left(t^{-1 / \alpha} s\right) \mathrm{d} s .
$$

The substitution $s=t^{1 / \alpha} u$ gives

$$
\begin{aligned}
& p(t, x, y) \\
& \geqslant C_{1} \mathrm{e}^{m t} \int_{0}^{\infty}\left(t^{1 / \alpha} u\right)^{-d / d_{w}} \mathrm{e}^{-C_{2}\left(t^{1 / \alpha} u\right)^{\frac{-1}{d_{w}-1}} \rho(x, y)^{\frac{d w}{d w-1}}} \mathrm{e}^{-m^{1 / \alpha} t^{1 / \alpha} u} \eta_{1}(u) \mathrm{d} u .
\end{aligned}
$$

Now, from 2.7 it follows that there exist $\delta(\alpha)>0$ and $c_{1}>0$ such that for all $s \leqslant \delta(\alpha)$,

$$
\eta_{1}(s) \geqslant c_{1} s^{-a_{1}} \mathrm{e}^{-a_{2} s^{-\alpha /(1-\alpha)}} .
$$

For further use, observe also that from $(2.8)$ it follows that there exist $u_{0}(\alpha)>0$ and $c_{2}>0$ such that for all $s \geqslant u_{0}(\alpha)$,

$$
\eta_{1}(s) \geqslant c_{2} s^{-1-\alpha} \text {. }
$$

CASE 1 . Assume $\rho(x, y) \geqslant t$, so that

$$
\min \left(\rho(x, y),\left(\rho(x, y) t^{-1 / d_{w}}\right)^{\frac{d_{w}}{d_{w}-1}}\right)=\rho(x, y) .
$$

Denote $A_{t}(x, y):=t^{-1 / \alpha} \rho(x, y)$.

- If $A_{t}(x, y) \leqslant \delta(\alpha)$ then we restrict the integration in 3.3 to the interval $\left(A_{t}(x, y) / 2, A_{t}(x, y)\right)$ to get

$$
\begin{gathered}
p(t, x, y) \\
\geqslant C_{1} \mathrm{e}^{m t} \int_{A_{t}(x, y) / 2}^{A_{t}(x, y)}\left(t^{1 / \alpha} u\right)^{-d / d_{w}} \mathrm{e}^{-C_{2}\left(t^{1 / \alpha} u\right)^{\frac{-1}{d w-1}} \rho(x, y)^{\frac{d_{w}}{d w-1}}-m^{1 / \alpha} t^{1 / \alpha} u} \eta_{1}(u) \mathrm{d} u \\
\geqslant c \mathrm{e}^{m t} \rho(x, y)^{-d / d_{w}} \mathrm{e}^{-c^{\prime} \rho(x, y)} \int_{A_{t}(x, y) / 2}^{A_{t}(x, y)} \eta_{1}(u) \mathrm{d} u,
\end{gathered}
$$


as the functions $f_{1}(s)=s^{-d / d_{w}}$ and $f_{2}(s)=\exp \left\{-m^{1 / \alpha} t^{1 / \alpha} s\right\}$ are decreasing and $f_{3}(s)=\exp \left\{-C_{2} s^{-1 /\left(d_{w}-1\right)} \rho(x, y)^{d_{w} /\left(d_{w}-1\right)}\right\}, s \in(0, \infty)$, is increasing. Using (3.4) and rearranging we get

$p(t, x, y)$

$$
\geqslant c \mathrm{e}^{m t} \rho(x, y)^{-d / d_{w}} \mathrm{e}^{-c^{\prime} \rho(x, y)}\left(t^{-1 / \alpha} \rho(x, y)\right)^{-a_{1}+1} \mathrm{e}^{-2^{\frac{\alpha}{\alpha-1}} a_{2} t^{\frac{1}{1-\alpha}} \rho(x, y)^{-\frac{\alpha}{1-\alpha}}}
$$

with some $c, c^{\prime}>0$. Note that for $\rho(x, y) \geqslant t$ we have

$$
\mathrm{e}^{-a_{2} t^{\frac{1}{1-\alpha}} \rho(x, y)^{-\frac{\alpha}{1-\alpha}}} \geqslant \mathrm{e}^{-a_{2} \rho(x, y)^{\frac{1}{1-\alpha}} \rho(x, y)^{-\frac{\alpha}{1-\alpha}}}=\mathrm{e}^{-a_{2} \rho(x, y)}
$$

so that

$$
p(t, x, y) \geqslant c t^{-d / d_{w}}\left(t^{d / d_{w}+a_{1} / \alpha-1 / \alpha} \mathrm{e}^{m t}\right)\left(\rho(x, y)^{-d / d_{w}-a_{1}+1} \mathrm{e}^{-c_{3} \rho(x, y)}\right) .
$$

It is clear that there exist constants $c_{4}, c_{5}>0$ independent of $x, y, t$ (recall that $\rho(x, y) \geqslant t \geqslant 1)$ such that $t^{d / d_{w}+a_{1}(\alpha) / \alpha-1 / \alpha} \mathrm{e}^{m t} \geqslant c_{4}$ and

$$
\rho(x, y)^{-d / d_{w}-a_{1}(\alpha)+1} \mathrm{e}^{-c_{3} \rho(x, y)} \geqslant c_{5} \mathrm{e}^{-2 c_{3} \rho(x, y)} .
$$

This is because for $p \in \mathbb{R}$ and $\mu \in \mathbb{R}_{+}$we have

$$
\inf _{\xi \geqslant 1} \xi^{p} \mathrm{e}^{\mu \xi}=c(p, \mu)>0 .
$$

This proves that

$$
p(t, x, y) \geqslant c t^{-d / d_{w}} \mathrm{e}^{-c^{\prime \prime} \rho(x, y)} \quad \text { for } \rho(x, y) \geqslant t \geqslant 1
$$

- If, on the other hand, $A_{t}(x, y)>\delta(\alpha)$, then also $B_{t}(x, y):=\frac{u_{0}(\alpha)}{\delta(\alpha)} A_{t}(x, y)$ $>u_{0}(\alpha)$. Now the integration in 3.3 will be restricted to the interval $\left(B_{t}(x, y), 2 B_{t}(x, y)\right)$. This gives, as before,

$$
\begin{aligned}
& p(t, x, y) \\
& \geqslant C_{1} \mathrm{e}^{m t} \int_{B_{t}(x, y)}^{2 B_{t}(x, y)}\left(t^{1 / \alpha} u\right)^{-d / d_{w}} \mathrm{e}^{-C_{2}\left(t^{1 / \alpha} u\right)^{\frac{-1}{d w-1}} \rho(x, y)^{\frac{d_{w}}{d w-1}}-m^{1 / \alpha} t^{1 / \alpha} u} \eta_{1}(u) \mathrm{d} u \\
& \geqslant c \mathrm{e}^{m t} \rho(x, y)^{-d / d_{w}} \mathrm{e}^{-c^{\prime} \rho(x, y)} \int_{B_{t}(x, y)}^{2 B_{t}(x, y)} \eta_{1}(u) \mathrm{d} u,
\end{aligned}
$$

and now from 3.5 it follows that

$$
\begin{aligned}
p(t, x, y) & \geqslant c \mathrm{e}^{m t} \rho(x, y)^{-d / d_{w}} \mathrm{e}^{-c^{\prime} \rho(x, y)}\left(t^{-1 / \alpha} \rho(x, y)\right)^{-\alpha} \\
& =c t^{-d / d_{w}}\left(t^{d / d_{w}+1} \mathrm{e}^{m t}\right)\left(\rho(x, y)^{-d / d_{w}-\alpha} \mathrm{e}^{-c^{\prime} \rho(x, y)}\right),
\end{aligned}
$$


which gives, in view of (3.6),

$$
p(t, x, y) \geqslant c t^{-d / d_{w}} \mathrm{e}^{-c^{\prime \prime} \rho(x, y)},
$$

as needed.

CASE 2. Assume now that $\rho(x, y)<t$, i.e.

$$
\min \left(\rho(x, y),\left(\rho(x, y) t^{-1 / d_{w}}\right)^{\frac{d_{w}}{d_{w}-1}}\right)=\left(\rho(x, y) t^{-1 / d_{w}}\right)^{\frac{d_{w}}{d_{w}-1}} .
$$

To obtain the bound (3.2) it is sufficient to show that there exist constants $L_{1}=$ $L_{1}(\alpha, m)>0, L_{2}=L_{2}(\alpha, m)>0, L_{1}<L_{2}$ such that

$$
\int_{L_{1} t}^{L_{2} t} \mathrm{e}^{-m^{1 / \alpha} s} \eta_{t}(s) \mathrm{d} s \geqslant c \mathrm{e}^{-m t} \quad \text { for some } c>0 .
$$

Indeed, in that case

$$
\begin{aligned}
p(t, x, y) & \geqslant C_{1} \mathrm{e}^{m t} \int_{L_{1} t}^{L_{2} t} s^{-d / d_{w}} \mathrm{e}^{-C_{2} s^{\frac{-1}{d_{w}-1}} \rho(x, y)^{\frac{d_{w}}{d_{w}-1}}} \mathrm{e}^{-m^{1 / \alpha} s} \eta_{t}(s) \mathrm{d} s \\
& \geqslant c \mathrm{e}^{m t} t^{-d / d_{w}} \mathrm{e}^{-c^{\prime} t^{\frac{-1}{d_{w}-1}} \rho(x, y)^{\frac{d_{w}}{d_{w}-1}}} \int_{L_{1} t}^{L_{2} t} \mathrm{e}^{-m^{1 / \alpha} s} \eta_{t}(s) \mathrm{d} s \\
& \geqslant c t^{-d / d_{w}} \mathrm{e}^{-c^{\prime \prime} t^{\frac{-1}{d_{w}-1}} \rho(x, y)^{\frac{d_{w}}{d_{w}-1}}} .
\end{aligned}
$$

Now we are going to show how to find $L_{1}$ and $L_{2}$. To this end, we will repeatedly use (2.5). For any $0<L_{1}<L_{2}<\infty$ we have

$$
\begin{aligned}
\mathrm{e}^{-m t} & =\int_{0}^{\infty} \mathrm{e}^{-m^{1 / \alpha} s} \eta_{t}(s) \mathrm{d} s \\
& =\int_{0}^{L_{1} t} \mathrm{e}^{-m^{1 / \alpha} s} \eta_{t}(s) \mathrm{d} s+\int_{L_{1} t}^{L_{2} t} \mathrm{e}^{-m^{1 / \alpha} s} \eta_{t}(s) \mathrm{d} s+\int_{L_{2} t}^{\infty} \mathrm{e}^{-m^{1 / \alpha} s} \eta_{t}(s) \mathrm{d} s .
\end{aligned}
$$

Note that

$$
\int_{0}^{L_{1} t} \mathrm{e}^{-m^{1 / \alpha} s} \eta_{t}(s) \mathrm{d} s=\int_{0}^{L_{1} t} \mathrm{e}^{s} \mathrm{e}^{-\left(m^{1 / \alpha}+1\right) s} \eta_{t}(s) \mathrm{d} s \leqslant \mathrm{e}^{L_{1} t} \mathrm{e}^{-t\left(m^{1 / \alpha}+1\right)^{\alpha}} ;
$$

let $\delta_{0}(\alpha)>0$ be defined by $\left(m^{1 / \alpha}+1\right)^{\alpha}=m+\delta_{0}(\alpha)$. Choose

$$
L_{1}:=\frac{1}{2} \delta_{0}(\alpha)
$$

Consequently,

$$
\int_{0}^{L_{1} t} \mathrm{e}^{-m^{1 / \alpha} s} \eta_{t}(s) \mathrm{d} s \leqslant \mathrm{e}^{-t\left(m+\frac{1}{2} \delta_{0}(\alpha)\right)}
$$


Also, note that for any $L_{2}>L_{1}$,

$$
\int_{L_{2} t}^{\infty} \mathrm{e}^{-m^{1 / \alpha} s} \eta_{t}(s) \mathrm{d} s \leqslant \mathrm{e}^{-m^{1 / \alpha} L_{2} t} \int_{L_{2} t}^{\infty} \eta_{t}(s) \mathrm{d} s \leqslant \mathrm{e}^{-m^{1 / \alpha} L_{2} t} .
$$

Inserting 3.11) and 3.12) into 3.10) we get

$$
\begin{aligned}
\int_{L_{1} t}^{L_{2} t} \mathrm{e}^{-m^{1 / \alpha} s} \eta_{t}(s) \mathrm{d} s & \geqslant \mathrm{e}^{-m t}-\mathrm{e}^{-t\left(m+\frac{1}{2} \delta_{0}(\alpha)\right)}-\mathrm{e}^{-m^{1 / \alpha} L_{2} t} \\
& =\mathrm{e}^{-m t}\left(1-\mathrm{e}^{-\frac{1}{2} \delta_{0}(\alpha) t}-\mathrm{e}^{\left(m-m^{1 / \alpha} L_{2}\right) t}\right) .
\end{aligned}
$$

For any $L_{2}>m^{1-1 / \alpha}$, we find that the function $t \mapsto 1-\mathrm{e}^{-\frac{1}{2} \delta_{0}(\alpha) t}-\mathrm{e}^{\left(m-m^{1 / \alpha} L_{2}\right) t}$ is increasing for $t \geqslant 1$, thus

$$
\int_{L_{1} t}^{L_{2} t} \mathrm{e}^{-m^{1 / \alpha} s} \eta_{t}(s) \mathrm{d} s \geqslant \mathrm{e}^{-m t}\left(1-\mathrm{e}^{-\frac{1}{2} \delta_{0}(\alpha)}-\mathrm{e}^{m-m^{1 / \alpha} L_{2}}\right),
$$

and in order to get 3.9$)$, it is sufficient to find $L_{2}>\max \left(L_{1}, m^{1-1 / \alpha}\right)$ for which

$$
1-\mathrm{e}^{-\frac{1}{2} \delta_{0}(\alpha)}-\mathrm{e}^{m-m^{1 / \alpha} L_{2}}>0 .
$$

This is equivalent to $L_{2}>\left(m-\ln \left(1-\mathrm{e}^{-\frac{1}{2} \delta_{0}(\alpha)}\right)\right) m^{-1 / \alpha}$, so finally we take

$$
L_{2}:=\max \left(L_{1}, m^{1-1 / \alpha}, \frac{m-\ln \left(1-\mathrm{e}^{-\delta_{0}(\alpha) / 2}\right)}{m^{1 / \alpha}}\right)+1 .
$$

II. The upper bound. We now prove that there exist constants $K_{3}=K_{3}(\alpha)$, $K_{4}=K_{4}(\alpha)>0$ such that for any $x, y \in F$ and $t \geqslant 1$,

$$
p(t, x, y) \leqslant K_{3} t^{-d / d_{w}} \mathrm{e}^{-K_{4} \min \left(\rho(x, y),\left(\rho(x, y) t^{-1 / d_{w}}\right)^{\frac{d_{w}}{d_{w}-1}}\right)} .
$$

Clearly, using (2.1) we can write

$$
p(t, x, y) \leqslant \mathrm{e}^{m t} \int_{0}^{\infty} C_{3} s^{-d / d_{w}} \mathrm{e}^{-C_{4} s^{\frac{-1}{d_{w}-1}} \rho(x, y)^{\frac{d_{w}}{d_{w}-1}}} \mathrm{e}^{-m^{1 / \alpha}} \eta_{t}(s) \mathrm{d} s .
$$

Case $\rho(x, y) \geqslant t$. Denote the integrand in 3.14 by $h(s)$, and consider the function

$$
f(s)=s^{-d / d_{w}} \mathrm{e}^{-C_{4} s^{\frac{-1}{d w-1}} \rho(x, y)^{\frac{d w}{d w-1}}}, \quad s \in(0, \infty),
$$

so that $h(s)=C_{3} f(s) \mathrm{e}^{-m^{1 / \alpha} s} \eta_{t}(s)$. We have

$$
\lim _{s \rightarrow 0^{+}} f(s)=\lim _{s \rightarrow \infty} f(s)=0 .
$$


By computing the derivative we check that $f(\cdot)$ is increasing on $\left(0, c_{6} \rho(x, y)^{d_{w}}\right)$ and decreasing on $\left(c_{6} \rho(x, y)^{d_{w}}, \infty\right)$, for some $c_{6}>0$. We now set

$$
c_{7}=\max \left(2 c_{6}, m^{1-1 / \alpha}+1\right) .
$$

We split the integral in 3.14 as follows:

$$
p(t, x, y) \leqslant \mathrm{e}^{m t}\left(\int_{0}^{c_{6} \rho(x, y)} h(s) \mathrm{d} s+\int_{c_{6} \rho(x, y)}^{c_{7} \rho(x, y)} h(s) \mathrm{d} s+\int_{c_{7} \rho(x, y)}^{\infty} h(s) \mathrm{d} s\right)
$$

Observing the monotonicity of the functions

$$
s \mapsto f(s), \quad s \mapsto s^{-d / d_{w}}, \quad s \mapsto \mathrm{e}^{-C_{4} s^{\frac{-1}{d_{w}-1}} \rho(x, y)^{\frac{d_{w}}{d_{w}-1}}},
$$

recalling 2.2 , and using $\rho(x, y) \geqslant t$, we get

$$
\begin{aligned}
\int_{0}^{c_{6} \rho(x, y)} h(s) \mathrm{d} s & \leqslant c \rho(x, y)^{-d / d_{w}} \mathrm{e}^{-c^{\prime} \rho(x, y)} \int_{0}^{c_{6} \rho(x, y)} \mathrm{e}^{-m^{1 / \alpha} s} \eta_{t}(s) \mathrm{d} s \\
& \leqslant c t^{-d / d_{w}} \mathrm{e}^{-m t} \mathrm{e}^{-c^{\prime} \rho(x, y)} ; \\
\int_{c_{6} \rho(x, y)}^{c_{7} \rho(x, y)} h(s) \mathrm{d} s & \leqslant c \rho(x, y)^{-d / d_{w}} \mathrm{e}^{-c^{\prime} \rho(x, y)} \int_{c_{6} \rho(x, y)}^{c_{7} \rho(x, y)} \mathrm{e}^{-m^{1 / \alpha} s} \eta_{t}(s) \mathrm{d} s \\
& \leqslant c t^{-d / d_{w}} \mathrm{e}^{-m t} \mathrm{e}^{-c^{\prime} \rho(x, y) .}
\end{aligned}
$$

We estimate the remaining integral as follows, recalling that $\int_{0}^{\infty} \eta_{t}(s) \mathrm{d} s=1$ :

$$
\begin{gathered}
\int_{c_{7} \rho(x, y)}^{\infty} C_{3} s^{-d / d_{w}} \mathrm{e}^{-C_{4} s^{\frac{-1}{d_{w}^{-1}}} \rho(x, y)^{\frac{d_{w}}{d_{w}-1}}} \mathrm{e}^{-m^{1 / \alpha}} \eta_{t}(s) \mathrm{d} s \\
\leqslant c \rho(x, y)^{-d / d_{w}} \int_{c_{7} \rho(x, y)}^{\infty} \mathrm{e}^{-m^{1 / \alpha} s} \eta_{t}(s) \mathrm{d} s \\
\leqslant c t^{-d / d_{w}} \mathrm{e}^{-m^{1 / \alpha}\left(m^{1-1 / \alpha}+1\right) \rho(x, y)} \int_{c_{7} \rho(x, y)}^{\infty} \eta_{t}(s) \mathrm{d} s \\
\leqslant c t^{-d / d_{w}} \mathrm{e}^{-m \rho(x, y)} \mathrm{e}^{-m^{1 / \alpha} \rho(x, y)} \leqslant c t^{-d / d_{w}} \mathrm{e}^{-m t} \mathrm{e}^{-m^{1 / \alpha} \rho(x, y)},
\end{gathered}
$$

which means that for $\rho(x, y) \geqslant t$, estimate 3.13 holds.

CASE $\rho(x, y)<t$. To begin, we define some constants:

$$
\begin{array}{ll}
k_{1}=(2 m)^{-1 / \alpha}, & k_{2}=\min \left(\frac{1}{2} k_{3}, k_{1}\right), \\
k_{3}=\frac{1}{2} \cdot \alpha \cdot m^{1-1 / \alpha}, & k_{4}=2 \max \left(k_{3}, m^{1-1 / \alpha}\right) .
\end{array}
$$


Then $k_{2} \leqslant k_{1}$ and $k_{2} \leqslant k_{3} t \leqslant k_{4} t$. With $h(s)$ as defined before,

$$
p(t, x, y) \leqslant \mathrm{e}^{m t}\left(\int_{0}^{k_{1}} h(s) \mathrm{d} s+\int_{k_{2}}^{k_{3} t} h(s) \mathrm{d} s+\int_{k_{3} t}^{k_{4} t} h(s) \mathrm{d} s+\int_{k_{4} t}^{\infty} h(s) \mathrm{d} s\right)
$$

Note, once $\rho(x, y)<t$, then also $k_{4} t^{\frac{-1}{d_{w}-1}} \rho(x, y)^{\frac{d_{w}}{d_{w}-1}} \leqslant k_{4} t$. Since the function $t \mapsto \mathrm{e}^{-C_{4} t^{\frac{-1}{d_{w}-1}} \rho(x, y)^{\frac{d_{w}}{d w}-1}}$ is nondecreasing, we can estimate the last integral in (3.15) as follows:

$$
\begin{gathered}
\int_{k_{4} t}^{\infty} C_{3} s^{-d / d_{w}} \mathrm{e}^{-C_{4} s^{\frac{-1}{d w^{-1}}} \rho(x, y)^{\frac{d_{w}}{d w-1}}} \mathrm{e}^{-m^{1 / \alpha} s} \eta_{t}(s) \mathrm{d} s \\
\leqslant c t^{-d / d_{w}} \int_{k_{4} t}^{\infty} \mathrm{e}^{-\frac{1}{2} m^{1 / \alpha} s} \eta_{t}(s) \mathrm{d} s \\
\leqslant c t^{-d / d_{w}} \mathrm{e}^{-m t} \mathrm{e}^{-c^{\prime} m^{1 / \alpha} t^{\frac{-1}{d_{w}-1}} \rho(x, y)^{\frac{d_{w}}{d_{w}-1}}} \int_{k_{4} t}^{\infty} \eta_{t}(s) \mathrm{d} s \\
\leqslant c \mathrm{e}^{-m t} t^{-d / d_{w}} \mathrm{e}^{-c^{\prime} m^{1 / \alpha} t^{\frac{-1}{d_{w}-1}} \rho(x, y)^{\frac{d_{w}}{d w-1}}} .
\end{gathered}
$$

For the previous integral we have

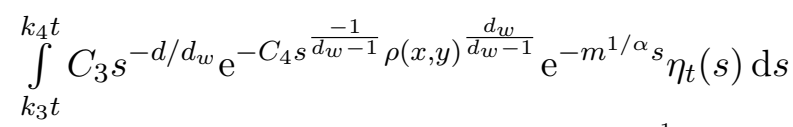

$$
\begin{aligned}
& \leqslant c t^{-d / d_{w}} \mathrm{e}^{-c^{\prime} t^{\frac{-1}{d_{w}-1}} \rho(x, y)^{\frac{d_{w}}{d w}-1}} \int_{k_{3} t}^{k_{4} t} \mathrm{e}^{-m^{1 / \alpha} s} \eta_{t}(s) \mathrm{d} s \\
& \leqslant c t^{-d / d_{w}} \mathrm{e}^{-c^{\prime} t^{\frac{-1}{d_{w}-1}} \rho(x, y)^{\frac{d_{w}}{d_{w}-1}}} \mathrm{e}^{-m t} .
\end{aligned}
$$

For the integral over $\left[0, k_{1}\right)$, using 2.9 , we get

$$
\begin{aligned}
& \int_{0}^{k_{1}} h(s) \mathrm{d} s \\
& \quad \leqslant c \mathrm{e}^{-C_{4}\left(k_{1} t\right)^{\frac{-1}{d w-1}} \rho(x, y)^{\frac{d w}{d w}-1}} \int_{0}^{k_{1}} s^{-d / d_{w}} \mathrm{e}^{-m^{1 / \alpha} s} t s^{-1-\alpha} \mathrm{e}^{-\frac{1}{2} t s^{-\alpha}} \mathrm{e}^{-\frac{1}{2} t s^{-\alpha}} \mathrm{d} s .
\end{aligned}
$$

The function $s \mapsto \mathrm{e}^{-\frac{1}{2} t s^{-\alpha}}$ is increasing, so that (recall the definition of $k_{1}$ )

$$
\begin{aligned}
& \int_{0}^{k_{1}} h(s) \mathrm{d} s \\
& \quad \leqslant c \mathrm{e}^{-m t} t^{-d / d_{w}} \mathrm{e}^{-c^{\prime} t^{\frac{-1}{d_{w}-1}} \rho(x, y)^{\frac{d_{w}}{d w}-1}} \int_{0}^{k_{1}} s^{-d / d_{w}-1-\alpha} \cdot 1 \cdot t^{d / d_{w}+1} \mathrm{e}^{-\frac{1}{2} t s^{-\alpha}} \mathrm{d} s .
\end{aligned}
$$


For the function $g_{s}(t)=t^{d / d_{w}+1} \mathrm{e}^{-\frac{1}{2} t s^{-\alpha}}, t \geqslant 1$, we have

$$
g_{s}(1)=\mathrm{e}^{-\frac{1}{2} s^{-\alpha}}, \quad \lim _{t \rightarrow \infty} g_{s}(t)=0,
$$

and also $g_{s}^{\prime}(t)=0 \Leftrightarrow t=c_{8} s^{\alpha}, c_{8}>0$. So, if $c_{8} s^{\alpha}<1$ then $\sup _{t \geqslant 1} g_{s}(t)=$ $g_{s}(1)=\mathrm{e}^{-\frac{1}{2} s^{-\alpha}}$, while if $c_{8} s^{\alpha} \geqslant 1$ then $\sup _{t \geqslant 1} g_{s}(t)=g_{s}\left(c_{8} s^{\alpha}\right)=c_{9} s^{\alpha d / d_{w}+\alpha}$.

Consequently, for any $t \geqslant 1$,

$$
\begin{aligned}
\int_{0}^{k_{1}} s^{-d / d_{w}-1-\alpha} t^{d / d_{w}+1} \mathrm{e}^{-\frac{1}{2} t s^{-\alpha}} \mathrm{d} s \leqslant & \int_{0}^{\min \left(c_{8}^{-1 / \alpha}, k_{1}\right)} s^{-d / d_{w}-1-\alpha} \mathrm{e}^{-\frac{1}{2} s^{-\alpha}} \mathrm{d} s \\
& +\int_{\min _{\left(c_{8}^{-1 / \alpha}, k_{1}\right)} c_{9} s^{-\left(d / d_{w}\right)(1-\alpha)-1} \mathrm{~d} s}^{k_{1}} \\
= & : M<\infty .
\end{aligned}
$$

We have obtained

$$
\int_{0}^{k_{1}} h(s) \mathrm{d} s \leqslant c \mathrm{e}^{-m t} t^{-d / d_{w}} \mathrm{e}^{-c^{\prime} t^{\frac{-1}{d w-1}} \rho(x, y)^{\frac{d_{w}}{d_{w}-1}}} .
$$

Finally, we are going to estimate $\int_{k_{2}}^{k_{3} t} h(s) \mathrm{d} s$. This estimate requires a finer analysis. Firstly, we point out that

$$
\lim _{\epsilon \rightarrow 0^{+}} \frac{2 \epsilon}{(1+3 \epsilon)^{1 / \alpha}-1}=\frac{2}{3} \alpha>\frac{1}{2} \alpha=m^{1 / \alpha-1} k_{3},
$$

so there exists $\epsilon_{0}(\alpha)>0$ such that

$$
\frac{2 \epsilon_{0}(\alpha)}{\left(1+3 \epsilon_{0}(\alpha)\right)^{1 / \alpha}-1} \geqslant m^{1 / \alpha-1} k_{3}
$$

We continue as follows:

$$
\mathrm{e}^{m t} \int_{k_{2}}^{k_{3} t} h(s) \mathrm{d} s \leqslant c t^{-d / d_{w}} \mathrm{e}^{-c^{\prime} t^{\frac{-1}{d_{w}-1}} \rho(x, y)^{\frac{d_{w}}{d w}-1}} \int_{k_{2}}^{k_{3} t} t^{d / d_{w}} \mathrm{e}^{m t} \mathrm{e}^{-m^{1 / \alpha} s} \eta_{t}(s) \mathrm{d} s .
$$

To prove 3.13 it is enough to show that

$$
I_{0}:=\int_{k_{2}}^{k_{3} t} t^{d / d_{w}} \mathrm{e}^{m t} \mathrm{e}^{-m^{1 / \alpha} s} \eta_{t}(s) \mathrm{d} s \leqslant K
$$

for some constant $K>0$ independent of $t \geqslant 1$. First, find $K_{0}>0$ such that for any $t \geqslant 1$,

$$
t^{d / d_{w}} \mathrm{e}^{m t} \leqslant K_{0} \mathrm{e}^{\left(1+\epsilon_{0}(\alpha)\right) m t},
$$

with $\epsilon_{0}(\alpha)$ taken from 3.16 . 
Let us write $\mathrm{e}^{-m^{1 / \alpha} s}$ in a somewhat different way:

$$
\mathrm{e}^{-m^{1 / \alpha} s}=\mathrm{e}^{-\left(1+3 \epsilon_{0}(\alpha)\right)^{1 / \alpha} m^{1 / \alpha} s} \mathrm{e}^{\left[\left(1+3 \epsilon_{0}(\alpha)\right)^{1 / \alpha}-1\right] m^{1 / \alpha} s} .
$$

Now, since the function $s \mapsto \mathrm{e}^{\left[\left(1+3 \epsilon_{0}(\alpha)\right)^{1 / \alpha}-1\right] m^{1 / \alpha} s}$ is increasing, we have

$$
I_{0} \leqslant K_{0} \mathrm{e}^{\left(1+\epsilon_{0}(\alpha)\right) m t} \mathrm{e}^{\left[\left(1+3 \epsilon_{0}(\alpha)\right)^{1 / \alpha}-1\right] m^{1 / \alpha}\left(k_{3} t\right)} \int_{k_{2}}^{k_{3} t} \mathrm{e}^{-\left(1+3 \epsilon_{0}(\alpha)\right)^{1 / \alpha} m^{1 / \alpha} s} \eta_{t}(s) \mathrm{d} s,
$$

and using 2.2 we get

$$
I_{0} \leqslant K_{0} \mathrm{e}^{\left(1+\epsilon_{0}(\alpha)\right) m t} \mathrm{e}^{\left[\left(1+3 \epsilon_{0}(\alpha)\right)^{1 / \alpha}-1\right] m^{1 / \alpha}\left(k_{3} t\right)} \mathrm{e}^{-t\left(1+3 \epsilon_{0}(\alpha)\right) m},
$$

so that

$$
I_{0} \leqslant K_{0} \mathrm{e}^{-2 \epsilon_{0}(\alpha) m t} \mathrm{e}^{\left[\left(1+3 \epsilon_{0}(\alpha)\right)^{1 / \alpha}-1\right] m^{1 / \alpha} k_{3} t},
$$

and to finish the proof we need to show that the exponent is nonpositive, i.e.

$$
2 \epsilon_{0}(\alpha) m \geqslant\left[\left(1+3 \epsilon_{0}(\alpha)\right)^{1 / \alpha}-1\right] m^{1 / \alpha} k_{3} .
$$

This is clear in view of 3.16 . We have proved (3.13), therefore the proof of 3.1 ) is complete.

Now we will give estimates for small times. In the range $\rho(x, y)<1$ the result is similar to the estimate for an $\alpha$-stable process from [11]. When $\rho(x, y) \geqslant 1$, the bound is exponential. To shorten notation, following [11], we denote $d_{\alpha}=$ $d+\alpha d_{w}$.

THEOREM 3.2. Let $X_{t}$ be a relativistic $\alpha$-stable process on $F$ with density function $p(\cdot, \cdot, \cdot)$ given by 2.12). Let $t \in(0,1)$. Then there exist constants $K_{i}=$ $K_{i}(\alpha)>0, i=5, \ldots, 10$, such that:

(1) for $\rho(x, y) \geqslant 1$,

$$
K_{5} t \mathrm{e}^{-K_{6} \rho(x, y)} \leqslant p(t, x, y) \leqslant K_{7} t \mathrm{e}^{-K_{8} \rho(x, y)},
$$

(2) for $\rho(x, y)<1$,

$$
\begin{aligned}
K_{9} \min \left(\frac{t}{\rho(x, y)^{d_{\alpha}}}\right. & \left.t^{-d /\left(\alpha d_{w}\right)}\right) \\
& \leqslant p(t, x, y) \leqslant K_{10} \min \left(\frac{t}{\rho(x, y)^{d_{\alpha}}}, t^{-d /\left(\alpha d_{w}\right)}\right) .
\end{aligned}
$$

Proof. (1) Case $\rho(x, y) \geqslant 1$. The lower bound. From (2.6) and 2.8 , we have

$$
\eta_{t}(u) \geqslant c t u^{-1-\alpha} \quad \text { for } u>u_{0} t^{1 / \alpha}
$$


where $u_{0}$ is taken from 3.5 . Then

$$
\begin{aligned}
p(t, x, y) & \geqslant C_{1} \mathrm{e}^{m t} \int_{u_{0} t^{1 / \alpha}}^{\infty} s^{-d / d_{w}} \mathrm{e}^{-C_{2} s^{\frac{-1}{d_{w}-1}} \rho(x, y)^{\frac{d_{w}}{d_{w}-1}}} \mathrm{e}^{-m^{1 / \alpha} s} \eta_{t}(s) \mathrm{d} s \\
& \geqslant c t \int_{u_{0} t^{1 / \alpha}}^{\infty} s^{-d / d_{w}-1-\alpha} \mathrm{e}^{-C_{2} s^{\frac{-1}{d w-1}} \rho(x, y)^{\frac{d_{w}}{d w-1}}} \mathrm{e}^{-m^{1 / \alpha} s} \mathrm{~d} s .
\end{aligned}
$$

Since $t \in(0,1)$ and $\rho(x, y) \geqslant 1$ we have $u_{0} t^{1 / \alpha}<u_{0} \rho(x, y)$, so that

$$
\begin{aligned}
p(t, x, y) & \geqslant c t \int_{u_{0} \rho(x, y)}^{\infty} s^{-d / d_{w}-1-\alpha} \mathrm{e}^{-C_{2} s^{\frac{-1}{d w-1}} \rho(x, y)^{\frac{d_{w}}{d w-1}}} \mathrm{e}^{-m^{1 / \alpha} s} \mathrm{~d} s \\
& \geqslant c t \mathrm{e}^{-C_{2}\left(u_{0} \rho(x, y)\right)^{\frac{-1}{d w-1}} \rho(x, y)^{\frac{d_{w}}{d_{w}-1}}} \int_{u_{0} \rho(x, y)}^{\infty} s^{-d / d_{w}-1-\alpha} \mathrm{e}^{-m^{1 / \alpha} s} \mathrm{~d} s \\
& \geqslant c t \mathrm{e}^{-c^{\prime} \rho(x, y)} \int_{u_{0} \rho(x, y)}^{\infty} \mathrm{e}^{-c^{\prime \prime} s} \mathrm{~d} s=c t \mathrm{e}^{-c^{\prime \prime \prime} \rho(x, y)} .
\end{aligned}
$$

The upper bound. Using 2.9 we get

$$
p(t, x, y) \leqslant c t \int_{0}^{\infty} s^{-d / d_{w}-1-\alpha} \mathrm{e}^{-C_{4} s^{\frac{-1}{d_{w}-1}} \rho(x, y)^{\frac{d_{w}}{d_{w}-1}}} \mathrm{e}^{-m^{1 / \alpha} s} \mathrm{~d} s .
$$

The function $s \mapsto s^{-d / d_{w}-1-\alpha} \mathrm{e}^{-C_{4} s^{\frac{-1}{d_{w}-1}} \rho(x, y)^{\frac{d w}{d w}-1}}$ is increasing on $\left(0, c_{10} \rho(x, y)\right)$, so that

$$
\begin{aligned}
& p(t, x, y) \leqslant c t\left(\int_{0}^{c_{10} \rho(x, y)} s^{-d / d_{w}-1-\alpha} \mathrm{e}^{-C_{4} s^{\frac{-1}{d_{w}-1}} \rho(x, y)^{\frac{d_{w}}{d_{w}-1}}} \mathrm{~d} s\right. \\
&\left.+\int_{c_{10} \rho(x, y)}^{\infty} s^{-d / d_{w}-1-\alpha} \mathrm{e}^{-m^{1 / \alpha} s} \mathrm{~d} s\right) \\
& \leqslant \leqslant c t\left(\rho(x, y)^{-d / d_{w}-\alpha} \mathrm{e}^{-c^{\prime} \rho(x, y)}+\rho(x, y)^{-d / d_{w}-1-\alpha} \int_{c_{10} \rho(x, y)}^{\infty} \mathrm{e}^{-m^{1 / \alpha} s} \mathrm{~d} s\right) \\
& \leqslant c t \mathrm{e}^{-c^{\prime \prime} \rho(x, y)},
\end{aligned}
$$

and the proof of 3.17$)$ is complete.

(2) Case $\rho(x, y)<1$. The upper bound. We have

$$
p(t, x, y)=\mathrm{e}^{m t} \int_{0}^{\infty} g(s, x, y) \mathrm{e}^{-m^{1 / \alpha} s} \eta_{t}(s) \mathrm{d} s \leqslant c \int_{0}^{\infty} g(s, x, y) \eta_{t}(s) \mathrm{d} s,
$$

which is the transition property of an $\alpha$-stable process, so from [11, Theorem 3.1] we get

$$
p(t, x, y) \leqslant K_{10} \min \left(t \rho(x, y)^{-d_{\alpha}}, t^{-\frac{d}{\alpha d_{w}}}\right) .
$$


The lower bound. To begin, note that

$$
t \rho(x, y)^{-d_{\alpha}} \leqslant t^{-\frac{d}{\alpha d_{w}}} \Longleftrightarrow t^{1 / \alpha} \leqslant \rho(x, y)^{d_{w}} .
$$

- If $t^{1 / \alpha} \leqslant \rho(x, y)^{d_{w}}$, then $u_{0} t^{1 / \alpha} \leqslant u_{0} \rho(x, y)^{d_{w}}$ and further

$$
\begin{aligned}
p(t, x, y) & \geqslant \mathrm{e}^{m t} \int_{u_{0} \rho(x, y)^{d} w}^{2 u_{0} \rho(x, y)^{d_{w}}} C_{1} s^{-d / d_{w}} \mathrm{e}^{-C_{2} s^{\frac{-1}{d_{w}-1}} \rho(x, y)^{\frac{d_{w}}{d_{w}-1}}} \mathrm{e}^{-m^{1 / \alpha} s} \eta_{t}(s) \mathrm{d} s \\
& \geqslant c \rho(x, y)^{-d} \int_{u_{0} \rho(x, y)^{d_{w}}}^{2 u_{0} \rho(x, y)^{d_{w}}} t s^{-1-\alpha} \mathrm{d} s \geqslant c t \rho(x, y)^{-d_{\alpha}} .
\end{aligned}
$$

- If $t^{1 / \alpha}>\rho(x, y)^{d_{w}}$, then

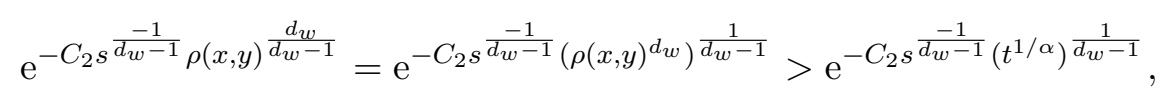

so

$$
\begin{aligned}
p(t, x, y) & \geqslant \mathrm{e}^{m t} \int_{u_{0} t^{1 / \alpha}}^{2 u_{0} t^{1 / \alpha}} C_{1} s^{-d / d_{w}} \mathrm{e}^{-C_{2} s^{\frac{-1}{d_{w}-1}}\left(t^{1 / \alpha}\right)^{\frac{1}{d_{w}-1}}} \mathrm{e}^{-m^{1 / \alpha} s} \eta_{t}(s) \mathrm{d} s \\
& \geqslant c t^{-\frac{d}{\alpha d_{w}}} \int_{u_{0} t^{1 / \alpha}}^{2 u_{0} t^{1 / \alpha}} t s^{-1-\alpha} \mathrm{d} s \geqslant c t^{-\frac{d}{\alpha d_{w}}}
\end{aligned}
$$

and the proof is complete.

As a corollary, we identify the domain of the Dirichlet form of a relativistic $\alpha$-stable process on $F$ and we provide estimates on the Dirichlet form itself.

Proposition 3.1. Let $(\mathcal{E}, \mathcal{D}(\mathcal{E}))$ be the Dirichlet form of a relativistic $\alpha$ stable process on $F$, with density function $p(\cdot, \cdot, \cdot)$ given by (2.12). Then

$$
\mathcal{D}(\mathcal{E})=\operatorname{Lip}\left(\alpha d_{w} / 2,2,2, F\right)
$$

and

$$
\begin{aligned}
\int_{F} \int_{F}(f(x)-f(y))^{2} & J_{1}(x, y) \mu(\mathrm{d} x) \mu(\mathrm{d} y) \\
& \leqslant \mathcal{E}(f, f) \leqslant \int_{F} \int_{F}(f(x)-f(y))^{2} J_{2}(x, y) \mu(\mathrm{d} x) \mu(\mathrm{d} y)
\end{aligned}
$$

where

$$
J_{i}(x, y)=K_{i, 1}\left(\rho(x, y)^{-d_{\alpha}} \mathbf{1}_{\{\rho(x, y)<1\}}+\mathrm{e}^{-K_{i, 2} \rho(x, y)} \mathbf{1}_{\{\rho(x, y) \geqslant 1\}}\right)
$$

for certain constants $K_{i, 1}, K_{i, 2}>0, i=1,2$. 
Proof. First observe that

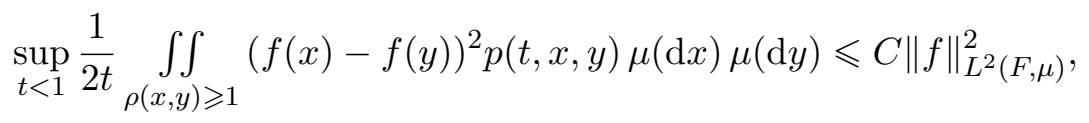

for both $\alpha$-stable and relativistic $\alpha$-stable processes.

Indeed, for a relativistic process, since $(f(x)-f(y))^{2} \leqslant 2\left(f(x)^{2}+f(y)^{2}\right)$, from symmetry and Fubini-Tonelli we have for any $f \in L^{2}(F, \mu)$ and $t<1$, using (3.17),

$$
\begin{aligned}
\frac{1}{2 t} \underset{\rho(x, y) \geqslant 1}{\iint_{f}(f(x)-f(y))^{2} p(t, x, y) \mu(\mathrm{d} x) \mu(\mathrm{d} y)} & \\
\leqslant & 2 K_{7} \int_{F} \int_{\{y: \rho(x, y) \geqslant 1\}} f(x)^{2} \mathrm{e}^{-K_{8} \rho(x, y)} \mu(\mathrm{d} y) \mu(\mathrm{d} x) \\
& =2 K_{7} \int_{F} f(x)^{2}\left(\int_{\rho(x, y) \geqslant 1} \mathrm{e}^{-K_{8} \rho(x, y)} \mu(\mathrm{d} y)\right) \mu(\mathrm{d} x) .
\end{aligned}
$$

Since $\mu$ is a $d$-measure, we can write

$$
\begin{aligned}
\int_{\rho(x, y) \geqslant 1} \mathrm{e}^{-K_{8} \rho(x, y)} \mu(\mathrm{d} y) & =\sum_{n=0}^{\infty} \int_{2^{n} \leqslant \rho(x, y)<2^{n+1}} \mathrm{e}^{-K_{8} \rho(x, y)} \mu(\mathrm{d} y) \\
& \leqslant c \sum_{n=0}^{\infty} \mathrm{e}^{-K_{8} 2^{n}} 2^{d n}<\infty
\end{aligned}
$$

(Cauchy condensation test). Consequently,

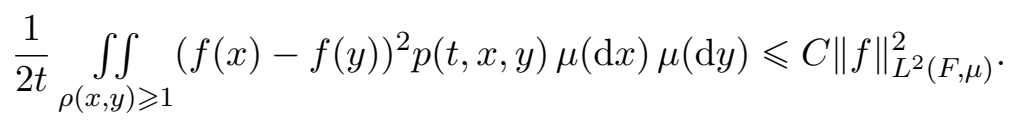

For an $\alpha$-stable process we proceed similarly, taking into account the fact that in that case the bound $(3.18)$ holds also for $\rho(x, y) \geqslant 1$.

Suppose now $f \in \operatorname{Lip}\left(\alpha d_{w} / 2,2,2, F\right)=\mathcal{D}\left(\mathcal{E}^{\text {stab }}\right)$. For a relativistic process we have (suppose $t<1$ )

$$
\begin{aligned}
& \mathcal{E}_{t}(f, f)=\frac{1}{2 t} \int_{F} \int_{F}(f(x)-f(y))^{2} p(t, x, y) \mu(\mathrm{d} x) \mu(\mathrm{d} y)
\end{aligned}
$$

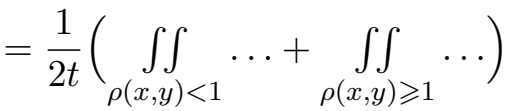

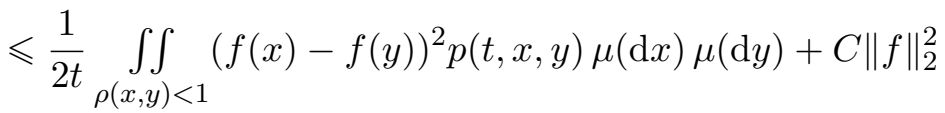

$$
\begin{aligned}
& \leqslant \mathcal{E}^{\mathrm{stab}}(f, f)+C\|f\|_{2}^{2},
\end{aligned}
$$

so that $f \in \mathcal{D}(\mathcal{E})$. The opposite inclusion is proven similarly. 
The formulas for $J_{1}(\cdot, \cdot), J_{2}(\cdot, \cdot)$ are easy consequences of 3.17 and 3.18. Assume $f \in \mathcal{D}(\mathcal{E})$. As above, we write

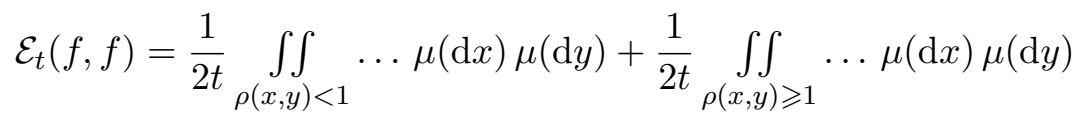

$$
\begin{aligned}
& =: \mathcal{E}_{t}^{(1)}(f, f)+\mathcal{E}_{t}^{(2)}(f, f) \text {. }
\end{aligned}
$$

The Dominated Convergence Theorem gives

$$
\begin{aligned}
\frac{1}{C} \underset{\rho(x, y) \leqslant 1}{\iint_{1}} \frac{(f(x)-f(y))^{2}}{\rho(x, y)^{d_{\alpha}}} \mu(\mathrm{d} x) \mu(\mathrm{d} y) \leqslant \liminf _{t \rightarrow 0} \mathcal{E}_{t}^{(1)}(f, f) \\
\quad \leqslant \limsup _{t \rightarrow 0} \mathcal{E}_{t}^{(1)}(f, f) \leqslant C \quad \iint_{\rho(x, y) \leqslant 1} \frac{(f(x)-f(y))^{2}}{\rho(x, y)^{d_{\alpha}}} \mu(\mathrm{d} x) \mu(\mathrm{d} y)
\end{aligned}
$$

for some $C>0$, and for the part $\mathcal{E}_{t}^{(2)}$ we have the straightforward estimates (recall $t<1)$

$$
\begin{aligned}
K_{5} \underset{\rho(x, y) \geqslant 1}{\iint_{\rho}(f(x)-} & f(y))^{2} \mathrm{e}^{-K_{6} \rho(x, y)} \mu(\mathrm{d} x) \mu(\mathrm{d} y) \leqslant \mathcal{E}_{t}^{(2)}(f, f) \\
& \leqslant K_{7} \underset{\rho(x, y) \geqslant 1}{\iint_{\rho}(f(x)-f(y))^{2} \mathrm{e}^{-K_{8} \rho(x, y)} \mu(\mathrm{d} x) \mu(\mathrm{d} y) .}
\end{aligned}
$$

The proof is complete.

\section{REFERENCES}

[1] M. T. Barlow, Diffusions on fractals, in: École d'Été de Probabilité de St. Flour XXV - 1995, Lecture Notes in Math. 1690, Springer, Berlin, 1998, 1-121.

[2] M. T. Barlow and R. F. Bass, The construction of Brownian motion on the Sierpiński carpet, Ann. Inst. H. Poincaré Probab. Statist. 25 (1989), 225-257.

[3] M. T. Barlow and R. F. Bass, Brownian motion and harmonic analysis on Sierpiński carpets, Canad. J. Math. 51 (1999), 673-744.

[4] M. T. Barlow, R. F. Bass, T. Kumagai, and A. Teplyaev, Uniqueness of Brownian motion on Sierpiński carpets, J. Eur. Math. Soc. 12 (2010), 655-701.

[5] M. T. Barlow and E. A. Perkins, Brownian motion on the Sierpiński gasket, Probab. Theory Related Fields 79 (1988), 543-623.

[6] J. Bertoin, Lévy Processes, Cambridge Univ. Press, Cambridge, 1996.

[7] J. Bertoin, Subordinators: examples and applications, in: École d'Été de Probabilités de St. Flour XXVII, P. Bernard (ed.), Lecture Notes in Math. 1717, Springer, 1999, 4-79.

[8] S. Bochner, Diffusion equation and stochastic processes, Proc. Nat. Acad. Sci. USA 35 (1949), 368-370.

[9] S. Bochner, Harmonic Analysis and the Theory of Probability, Univ. of California Press, Berkeley, CA, 1955.

[10] K. Bogdan et al., Potential Analysis of Stable Processes and its Extensions (ed. by P. Graczyk and A. Stós), Lecture Notes in Math. 1980, Springer, Berlin, 2009. 
[11] K. Bogdan, A. Stós, and P. Sztonyk, Harnack inequality for stable processes on d-sets, Studia Math. 158 (2003), 163-198.

[12] E. A. Carlen, S. Kusuoka, and D. W. Stroock, Upper bounds for symmetric Markov transition functions, Ann. Inst. H. Poincaré Probab. Statist. 23 (1987), 245-287.

[13] R. Carmona, W. C. Masters, and B. Simon, Relativistic Schrödinger operators: Asymptotic behaviour of the eigenfunctions, J. Funct. Anal. 91 (1990), 117-142.

[14] Z.-Q. Chen and T. Kumagai, Heat kernel estimates for stable-like processes on d-sets, Stoch. Processes Appl. 108 (2003), 27-62.

[15] Z.-Q. Chen and T. Kumagai, Heat kernel estimates for jump processes of mixed types on metric measure spaces, Probab. Theory Related Fields 140 (2008), 277-317.

[16] W. Farkas and N. Jacob, Sobolev spaces on non-smooth domains and Dirichlet forms related to subordinate reflecting diffusions, Math. Nachr. 224 (2001), 75-104.

[17] M. Fukushima, Dirichlet forms, diffusion processes, and spectral dimensions for nested fractals, in: Ideas and Methods in Mathematical Analysis, Stochastics, and Applications (Oslo, 1988), Cambridge Univ. Press., Cambridge, 1992, 151-161.

[18] M. Fukushima, Y. Oshima, and M. Takeda, Dirichlet Forms and Symmetric Markov Processes, de Gruyter, Berlin, 1994.

[19] A. Grigor'yan, Heat kernels and function theory on metric measure spaces, in: Heat Kernels and Analysis on Manifolds, Graphs, and Metric Spaces (Paris, 2002), Contemp. Math. 338, Amer. Math. Soc., Providence, RI, 2003, 143-172.

[20] A. Grigor'yan, H. Hu, and K.-S. Lau, Heat kernels on metric measure spaces and an application to semilinear elliptic equations, Trans. Amer. Math. Soc. 355 (2003), 2065-2095.

[21] S. Havlin and D. Ben-Avraham, Diffusion in disordered media, Adv. Phys. 36 (1987), 695-798.

[22] J. Hawkes, A lower Lipschitz condition for the stable subordinator, Z. Wahrsch. Verw. Gebiete 17 (1971), 23-32.

[23] M. Hino and T. Kumagai, A trace theorem for Dirichlet forms on fractals, J. Funct. Anal. 238 (2006), 578-611.

[24] J. Hu and M. Zähle, Potential spaces on fractals, Studia Math. 170 (2005), 259-281.

[25] J. Hu and M. Zähle, Generalized Bessel and Riesz potentials on metric measure spaces, Potential Anal. 30 (2009), 315-340.

[26] N. Jacob and R. Schilling, Some Dirichlet spaces obtained by subordinate reflected diffusions, Rev. Mat. Iberoamer. 15 (1999), 59-91.

[27] A. Jonsson, Brownian motion on fractals and function spaces, Math. Z. 222 (1996), 495-504.

[28] A. Jonsson, A trace theorem for the Dirichlet form on the Sierpinski gasket, Math. Z. 250 (2005), 599-609.

[29] A. Jonsson and H. Wallin, Function Spaces on Subsets of $\mathbb{R}^{n}$, Math. Reports 2, Part 1, Harwood, 1984.

[30] K. Kaleta and P. Sztonyk, Small-time sharp bounds for kernels of convolution semigroups, J. Anal. Math. 132 (2017), 355-394.

[31] P. Kim and A. Mimica, Green function estimates for subordinate Brownian motions: stable and beyond, Trans. Amer. Math. Soc. 366 (2014), 4383-4422.

[32] T. Kulczycki and B. Siudeja, Intrinsic ulrtacontractivity of the Feynman-Kac semigroup for relativistic stable processes, Trans. Amer. Math. Soc. 358 (2006), 5025-5057.

[33] T. Kumagai, Estimates on transition densities for Brownian motion on nested fractals, Probab. Theory Related Fields 96 (1993), 205-224.

[34] T. Kumagai, Function spaces and stochastic processes on fractals, in: Fractal Geometry and Stochastics III, Progr. Probab. 57, Birkhäuser, Basel, 2004, 221-234.

[35] E. H. Lieb and R. Seiringer, The Stability of Matter in Quantum Mechanics, Cambridge Univ. Press, 2009.

[36] T. Lindstrøm, Brownian motion on nested fractals, Mem. Amer. Math. Soc. 83 (1990), no. 420, iv $+128 \mathrm{pp}$.

[37] K. Pietruska-Pałuba, On function spaces related to the fractional diffusions on d-sets, Stoch. Stoch. Reports 70 (2000), 153-164. 
[38] M. Ryznar, Estimates of Green function for relativistic $\alpha$-stable process, Potential Anal. 17 (2002), 1-23.

[39] C. Sabot, Existence and uniqueness of diffusions on finitely ramified self-similar fractals, Ann. Sci. École Norm. Sup. (4) 30 (1997), 605-673.

[40] R. Schilling, R. Song and Z. Vondraček, Bernstein Functions. Theory and Applications, de Gruyter Stud. Math. 37, de Gruyter, Berlin, 2010.

[41] R. Schilling and J. Wang, Functional inequalities and subordination: stability of Nash and Poincaré inequalities, Math. Z. 272 (2012), 921-936.

[42] A. Stós, Symmetric $\alpha$-stable processes on d-sets, Bull. Polish Acad. Sci. Math. 48 (2000), 237-245.

[43] K.-T. Sturm, Diffusion processes and heat kernels on metric spaces, Ann. Probab. 26 (1998), $1-55$.

[44] H. Triebel, Theory of Function Spaces. III, Monogr. Math. 100, Birkhäuser, Basel, 2006.

[45] H. Triebel, Fractals and Spectra Related to Fourier Analysis and Function Spaces, Modern Birkhäuser Classics, Birkhäuser, Basel, 2011.

Hubert Balsam, Katarzyna Pietruska-Pałuba

Faculty of Mathematics, Informatics and Mechanics

University of Warsaw

Banacha 2

02-097 Warszawa, Poland

E-mail: h.balsam@mimuw.edu.pl

K.Pietruska-Paluba@mimuw.edu.pl

Received 24.10.2018;

revised version 12.2.2019 